\title{
Uric Acid in Cardiovascular Diseases: A Factor or an Incident?
}

\author{
LUCRETIA ANGHEL, ADRIAN BEZNEA*, ANCA-IULIA NEAGU, GABI TOPOR*, CRISTIAN ONISOR*, \\ SILVIA FOTEA \\ Dunarea de Jos University of Galați, Faculty of Medicine and Pharmacy, 47 Domnească Str., 800008, Galati, Romania
}

\begin{abstract}
The pathophysiological involvement of uric acid in cardiovascular disease has been intensively addressed in the last decades by the medical community. The tendencies to elucidate its role in cardiac events have been carried out in numerous researches with controversial results. In an attempt to understand this phenomenon, the existence of a paradox is admited, according to which uric acid behaves as an antioxidant, capturing reactive oxygen species, and in the context of an existing cardiac pathology, manifests a precipitating oxidative effect of circulating lipids, there being also present. inflammatory substrate. The involvement of the target organs in hypertension, along with other factors such as hyperlipidemia, obesity, pathologies of the valvular apparatus, contributes to the appearance of hyperuricemia of excretory cause. The ability of xanthine oxidoreductase to produce reactive oxygen species that contribute to the occurrence of oxidative stress and endothelial dysfunction has redirected our visions to a more complex approach to ischemic heart disease. And the use of xanthine oxidoreductase inhibitors in cardiovascular disease in patients with hyperuricemia has allowed to appreciate real benefits in improving renal function and preventing atherosclerosis.
\end{abstract}

Keywords: uric acid, cardiovascular disease, factor

Uric acid results from purine catabolism. In humans it is synthesized by xanthine dehydrogenase, otherwise mammals can subsequently be transformed into a more soluble compound - allantoin, for uricase [1]. Loss of the ability to synthesize ascorbic acid during evolution, an introductory urge to uric acid as an antioxidant component, replacing the L-gulonolacton oxidase gene by mutation, followed by uricase gene modification [2]. Although the relationship between ascorbic acid and uric acid is paradoxical, the first has been shown to reduce serum uric acid concentration [3]. The uric acid concentration varies from organ to organ, but there is an assumption that liver produce the largest amount, and excretion may be in the main in kidneys (70\%), and intestines. More than $60 \%$ of uric acid formed has as the main source endogenous purines, the rest are exogenous [4].

The physiological balance of uric acid level is maintained by enzymes involved in the synthesis, conversion and degradation of purine compounds: 5'-Nucleotidase, adenosine deaminase, xanthine oxidase (XO), purine nucleoside phosphorylase. The main factors that can alter this physiological balance, either directly or indirectly, are: genetic predisposition, obesity, alcohol consumption, thiazide and goat diuretics, hypothyroidism, hypertension, insulin resistance, renal dysfunction [5-7]. Hyperuricemia may be due to hyperproduction, diminished excretion, and mixed. Hyperproduction refers to increased meat consumption, tumor lysis syndrome, Lesch-Nyhan syndrome, KelleySeegmiller syndrome, increased phosphoribosyl pyrophosphate synthetase activity, increased turnover of nucleic acids (in hemolytic anemia, lymphomas, myelomas, leukemias). Decreased excretion involves renal dysfunction, the use of diuretics, salicylates, some anti-tuberculosis drugs. The mixed cause suggests starvation, alcohol consumption, high fructose diet..

În As an antioxidant, uric acid yields ascorbic acid and vitamin E, although some studies suggest its protective activity [8-10]. On the other hand, uric acid can have proinflammatory and oxidizing properties: urate interacts with peroxynitrite forming allantoin, aloxane as well as urate derivatives, accelerating LDL (low density lipoprotein) oxidation [11]. The pro-oxidant process occurs at the lipid level due to the inability to capture lipophilic radicals, although in a hydrophilic environment it exhibits antioxidant properties by capturing peroxyl radicals [12]. NADPH oxidase is involved in the proinflammatory process, which plays a role in cellular signaling, constituting a generator of reactive oxygen species.

Hyperuricemia is often associated with hypertension [13-15]. Also having a predictive role: adolescents with a high concentration of uric acid associate the risk of further development of hypertension [16], and the cardiovascular mortality rate is increased [17].

*email:adrianbeznea@hotmail.com,topor_gabi_atu@yahoo.com,cristi.onisor@gmail.com 


\section{Endothelial dysfunction}

There are conflicting assumptions regarding the action of uric acid on the vascular endothelium. Some studies [1820] suggest a beneficial effect, believing that increased serum levels are a response to oxidative stress, inflammatory substrate, in cardiovascular disease, and that uric acid actually represents a nitric oxide generator. On the other hand, other studies state that uric acid decreases nitric oxide levels by entering it in an irreversible reaction and forms 6aminouracil. Under oxidative stress, this reaction is partially blocked by another antioxidant - glutathione, and the nitric oxide level will not decrease drastically [21,22]. Therefore, the endothelium exhibits an impaired, decreased response to acetylcholine resulting in unsubstantial vasodilation. Also circulating platelets are involved in the process, which can precipitate endothelial dysfunction.

The high level of uric acid in the conditions of tissue ischemia due to heart failure and type 2 diabetes is due to the increased activity of XO that produces oxidants. In the case of treatment to reduce the serum uric acid concentration with allopurinol, it seems that the therapeutic benefit is more related to the decrease of oxidative stress [23,24].

\section{Atherosclerosis and uric acid}

The mechanism of atherosclerosis is based on chronic inflammation. Due to the turbulent blood flow in certain segments of the arterial system, gradual injury of the vascular intima occurs. Although there are protective factors such as the release by endothelial cells of matrix proteins and metalloproteinases for repair, prolonged stress on the intima causes activation of the NF- $\mathrm{kB}$ pathway that contributes to the formation of atherosclerotic lesion. Thinning of the glycocalyx following turbulent blood flow facilitates LDL lesion uptake and T lymphocyte infiltration. Following LDL oxidation of the lesion area, oxysterol formation occurs which has a negative effect on arterial elasticity expressed by: cytotoxicity, inflammation, apoptosis. In this context, uric acid can increase LDL oxidation by forming free radicals from urates. However, in the case of cardiovascular disease, the risk of occurrence is more hypertension and hyperlipidemia than hyperuricemia. Regarding the latter, most often it cannot be an individual risk factor [25-28].

\section{Heart failure}

Risk factors for heart failure are: male, obesity, hypertension, valvular dysfunction, coronary heart disease [29]. The correlation between hyperuricemia and heart failure can be achieved both directly and indirectly through other risk factors, uric acid reduction medication, as well as by combining all pathways and factors. Decreased renal circulation in heart failure serves to increase uric acid level by reducing its excretion. The mechanism of insufficient excretion and the administration of diuretics have common elements in the pathogenesis of hyperuricemia and heart failure, but they are also independent elements [27].

Cardiac remodeling may be affected by XO inhibition, and architectural reversibility in incipient heart failure can be achieved [30]. But it should be mentioned that not the low level of uric acid itself will contribute to this event but also other metabolic changes that occur in the myocardial cells, namely the reduction of oxidative stress. Low uric acid concentration will improve compromised renal function. The Framingham study also suggested that hyperuricemia is a risk factor for developing heart failure at an initial level> $6.4 \mathrm{mg} / \mathrm{dl}$ [31].

\section{Benefits of treatment with XO inhibitors}

For patients with gout, conventional treatment consists of XO inhibitors such as allopurinol and febuxostat, which are usually associated with an appropriate lifestyle and diet.

Allopurinol being a purine analog is metabolized by a variety of enzymes involved in the synthesis of purine and pyrimidine. While febuxostat is only an XO inhibitor, it does not disrupt the activity of other enzymes such as nucleoside phosphorylase and orotidin-5'-monophosphate decarboxylase, required in DNA and RNA synthesis [32]. One benefit of XO inhibitors is lower blood pressure in hypertensive patients. Thus, a trial whose participants were patients with hyperuricemia (> $7.0 \mathrm{mg} / \mathrm{dl}$ ) hypertension and normal renal function, by administering $80 \mathrm{mg}$ febuxostat daily, achieved a reduction of systolic blood pressure by 10 and $8 \mathrm{~mm} / \mathrm{Hg}$ dyastolic, respectively. [33]. The mechanism that contributed to the improvement of blood pressure would be the action of febuxostat on endothelial function, and renal protection against ischemia [34].

Also in the case of chronic kidney disease, hyperuricemia is a major risk factor for the development of the disease or the decline of glomerular filtration in the already established disease, and the treatment with short-term XO inhibitors being well tolerated indicates successes in preventing the deterioration of renal function [35,36 ].

\section{Conclusions}

Hyperuricemia in cardiovascular diseases is not an independent factor but rather correlates with others that have contributed to the development of the pathology: dyslipidemia, insulin resistance, use of diuretics, glucose intolerance. 
The mechanism by which XO inhibitors produce beneficial changes in the context of vascular diseases translates into the action of restoring endothelial function and reducing oxidative stress. And the reduction of uric acid level in asymptomatic cases does not seem to have any influence.

In order to confirm the long-term benefit of treatment with XO inhibitors, the existence of large-scale studies is imperative.

\section{References}

1.MAIUOLO J., et al. Regulation of uric acid metabolism and excretion, In International Journal of Cardiology. 2016;213:8-14.

2.DROUIN G., GODIN J., PAGÉ B. The genetics of vitamin C loss in vertebrates. In Curr Genomics. 2011;12(5):371-378.

3.JURASCHEK S.P., MILLER E.R., GELBER A.C. Effect of oral vitamin C supplementation on serum uric acid: a meta-analysis of randomized controlled trials. In Arthritis Care Res (Hoboken). 2011;63(9):1295-1306.

4.FREI B., STOCKER R., AMES B.N. Antioxidant defenses and lipid peroxidation in human blood plasma. Proc Natl Acad Sci USA. 1988;85:9748-9752.

5.YAMAMOTO T, MORIWAKI Y, TAKAHASHI S. Effect of ethanol on metabolism of purine bases (hypoxanthine, xanthine, and uric acid. Clinica Chimica Acta. In International Journal of Clinical Chemistry. 2005; 356(1-2): 35-57.

6.M. CHONCHOL, M.G. et al. Relationship of uric acid with progression of kidney disease. In Am J Kidney Dis. 2007;50:239-247.

7.ICHIDA K. et al. Decreased extra-renal urate excretion is a common cause of hyperuricemia. In Nat Commun. 2012;3:764-770.

8.HOSOMI A et al. Extra-renal elimination of uric acid via intestinal efflux transporter BCRP/ABCG2. In PLoS One. 2002;7(2):e30456.

9.ALVAREZ-LARIO B, J. MACARRON-VICENTE. Uric acid and evolution. In Rheumatology. 2010;49(11):2010-2016.

10. SPITSIN SV et al. Comparison of uric acid and ascorbic acid in protection against EAE. In Free Radic Biol Med. 2002;33:1363-1371.

11.SANTOS CX, ANJOS EI, AUGUSTO O. Uric acid oxidation by peroxynitrite: multiple reactions, free radical formation, and amplification of lipid oxidation. In Arch Biochem Biophys. 1999;372:285-294.

12.MURAOKA S, MIURA T. Inhibition by uric acid of free radicals that damage biological molecules. In Pharmacol Toxicol. 2003;93:284289.

13.JOHNSON R.J. et al. Is there a pathogenetic role for uric acid in hypertension and cardiovascular and renal disease? In Hypertension. 2003;41:1183-1190.

14.FANG J, MICHAEL H, ALDERMAN MH. Serum uric acid and cardiovascular mortality: the NHANES I epidemiologic follow up study, 1971-1992. In JAMA. 2000;28:2404-2410.

15.J.P. FORMAN, H. CHOI, G.C. CURHAN. Uric acid and insulin sensitivity and risk of incident hypertension. In Arch Intern Med. 2009;169:155-162

16.ALPER AB et al. Childhood uric acid predicts adult blood pressure: the Bogalusa Heart Study. In Hypertension. 2005;45:34-38.

17.TOMITA M et al. Does hyperuricemia affect mortality? A prospective cohort study of Japanese male workers. In J Epidemiol. 2000;10:403409.

18.NIETO FJ, et al Uric acid and serum antioxidant capacity: a reaction to atherosclerosis?

Atherosclerosis. 2000;148:131-139.

19.SUZUKI T. Nitrosation of uric acid induced by nitric oxide under aerobic conditions

Nitric Oxide. 2017;16:266-273.

20.SKINNER KA et al. Nitrosation of uric acid by peroxynitrite. Formation of a vasoactive nitric oxide donor. In J Biol Chem. 1998;273:2449124497.

21.GERSCH C et al. Inactivation of nitric oxide by uric acid. In Nucleosides Nucleotides Nucleic Acids. 2008;27:967-978.

22.KHOSLA UM et al. Hyperuricemia induces endothelial dysfunction In Kidney Int. 2005;67:1739-1742.

23.FARQUHARSON CA et al. Allopurinol improves endothelial dysfunction in chronic heart failure. In Circulation. 2002;106:221-226.

24.BUTLER R et al. Allopurinol normalizes endothelial dysfunction in type 2 diabetics with mild hypertension. In Hypertension.2000;35:746751.

25.NISHIOKA K, Iwatani M, Nihon Rinsho. Hyperuricemia and atherosclerosis. In Japanese Journal of Clinical Medicine.1993;51(8):21772181.

26.REUNANEN, A. et al. Hyperuricemia as a Risk Factor for Cardiovascular Mortality. In Acta Medica Scandinavica. 1982;212:49-59.

27.KIM, S. Y. et al. Hyperuricemia and coronary heart disease: A systematic review and meta- analysis. In Arthritis Care Res, 62: 170-180.

28.SOHA I et al. Association of asymptomatic hyperuricemia and endothelial dysfunction in psoriatic arthritis. In The Egyptian Rheumatologist. 2012;34(2)83-89.

29.KENCHAIAH S, NARULA J, VASAN RS. Risk factors for heart failure. In Med Clin North Am. 2004;88:1145-1172.

30.MINHAS KM et al. Xanthine oxidoreductase inhibition causes reverse remodeling in rats with dilated cardiomyopathy. In Circulation research. 2006;98:271-9.

31.CULLETON BF et al. Serum uric acid and risk for cardiovascular disease and death: the Framingham Heart Study. In Ann Intern Med. $1999 ; 131: 7-13$.

32.ERNST ME, FRAVEL MA. Febuxostat: a selective xanthine-oxidase/xanthine-dehydrogenase inhibitor for the management of hyperuricemia in adults with gout. In Clin Ther. 2009;31(11):2503-18.

33.LHANOO GUNAWARDHANA et al. Effect of Febuxostat on Ambulatory Blood Pressure in Subjects With Hyperuricemia and Hypertension: A Phase 2 Randomized Placebo- Controlled Study. In Journal of the American Heart Association. 2017;6:e006683.

34. TSUDA H et al. Febuxostat suppressed renal ischemia reperfusion injury via reduced oxidative stress. Biochem Biophys Res Commun. 2012; 427:266-272.

35. SIRCAR D et al. Efficacy of Febuxostat for Slowing the GFR Decline in Patients With CKD and Asymptomatic Hyperuricemia:A 6-Month, Double-Blind, Randomized, Placebo-Controlled Trial. In Am J Kidney Dis. 2015;66(6):945-50.

36. HOSOYA T et al. The effect of febuxostat to prevent a further reduction in renal function of patients with hyperuricemia who have never had gout and are complicated by chronic kidney disease stage 3: study protocol for a multicenter randomized controlled study. Trials. 2014;15:26.

Manuscript received: 19.11 .2019 